EPJ Web of Conferences 37, 09024 (2012)

DOI: 10.1051/epjconf/20123709024

C) Owned by the authors, published by EDP Sciences, 2012

\title{
Radial and angular-momentum Regge trajectories: a systematic approach
}

\author{
P. Masjuan ${ }^{1, a, b}$, E.R.Arriola ${ }^{2}$, and W. Broniowski $i^{3,4}$ \\ 1 Departamento de Física Teórica y del Cosmos and CAFPE, Universidad de Granada, E-18071 \\ Granada, Spain \\ 2 Departamento de Física Atómica, Molecular y Nuclear and Instituto Carlos I de Física Teórica y \\ Computacional, Universidad de Granada, E-18071 Granada, Spain \\ 3 The H. Niewodniczański Institute of Nuclear Physics, PL-31342 Kraków, Poland \\ 4 Institute of Physics, Jan Kochanowski University, PL-25406 Kielce, Poland
}

\begin{abstract}
We present the analysis of Ref. [1] of the radial ( $n$ ) and angular-momentum $(J)$ Regge trajectories for all light-quark meson states listed in the Particle Data Tables. The parameters of the trajectories are obtained with linear regression, with weight of each resonance inversely proportional to its half-width squared, $(\Gamma / 2)^{2}$. The joint analysis in the $\left(n, J, M^{2}\right)$ Regge plane indicates, at the 4.5 standard deviation level, that the slopes in $n$ are larger from the slopes in $J$. Thus no strict universality of slopes occurs in the light non-strange meson sector. We also extend our analysis to the kaon sector.
\end{abstract}

In Ref. [2] it was suggested that the light-quark meson states could be grouped into radial linear Regge trajectories with the slope $\mu^{2}=1.25(15) \mathrm{GeV}^{2}$, where the error was estimated as the spread of the values for each meson-family considered $(\rho, \pi, \eta, a, f)$. In Ref. [3] a joined formula assuming universality of slopes was proposed, $M^{2}(n, J)=b+a(n+J)$, with $a=1.14 \mathrm{GeV}^{2}$. In Ref. [1] we reanalyzed the radial and angular-momentum Regge trajectories with the updated list of the light unflavored mesons from the PDG [4]. For the fits and error estimates we have used the half-widht rule $[1,5]$, i.e, the half-widht squared as a weight for each resonance.

The fit to all the light unflavored meson families with linear trajectories using the half-width rule yields $\mu^{2}=1.35(4) \mathrm{GeV}^{2}$ as the weighted averaged result for the slope of radial trajectories, and $\beta^{2}=1.16(4) \mathrm{GeV}^{2}$ as the weighted average for the slope of the angular-momentum trajectories (the bands in Fig. 2). Fig. 1 exemplifies the results for the $\eta$ and $\rho$ families. In Fig. 2, we collect the slopes from both radial and angular-momentum trajectories from all the families considered.

We also considered a joint fit with the formula $M_{X}^{2}(n, J)=M_{X}^{2}(0,0)+n \mu^{2}+J \beta^{2}$, with the result $M_{X}^{2}(n, J)=(-1.25(4)+1.38(4) n+1.12(4) J) \mathrm{GeV}^{2}$, which means a difference between the radial and the angular-momentum slopes at a statistically significant level of 4.5 standard deviations.

As an extension of Ref. [1], we present in Fig. 3 a study of both radial and angular-momentum trajectories for the kaon sector. The radial fit yields $\mu_{K}^{2}=1.22(21) \mathrm{GeV}^{2}$ and $1.12(21) \mathrm{GeV}^{2}$ for $K$ and $K^{*}$, respectively, while the angular-momentum fit returns $\beta_{K}^{2}=1.36(6) \mathrm{GeV}^{2}$ and 1.19(7) $\mathrm{GeV}^{2}$ for $K$ and $K^{*}$, respectively. Only trajectories containing more than three states are considered.

\footnotetext{
a e-mail: masjuan@ugr.es
}

b Supported by MICINN of Spain (FPA2006-05294), CPAN (CSD2007- 00042), Junta de Andalucía (FQM 101, FQM 437, FQM 225, and FQM 022), by the Deutsche Forschungsgemeinschaft DFG through the Collaborative Research Center “The Low-Energy Frontier of the Standard Model” (SFB 1044), by Polish Ministry of Science and Higher Education, grant N N202 263438, and by National Science, grant DEC-2011/01/D/ST2/00772.

This is an Open Access article distributed under the terms of the Creative Commons Attribution License 2.0, which permits unrestricted use, distribution, and reproduction in any medium, provided the original work is properly cited. 
EPJ Web of Conferences
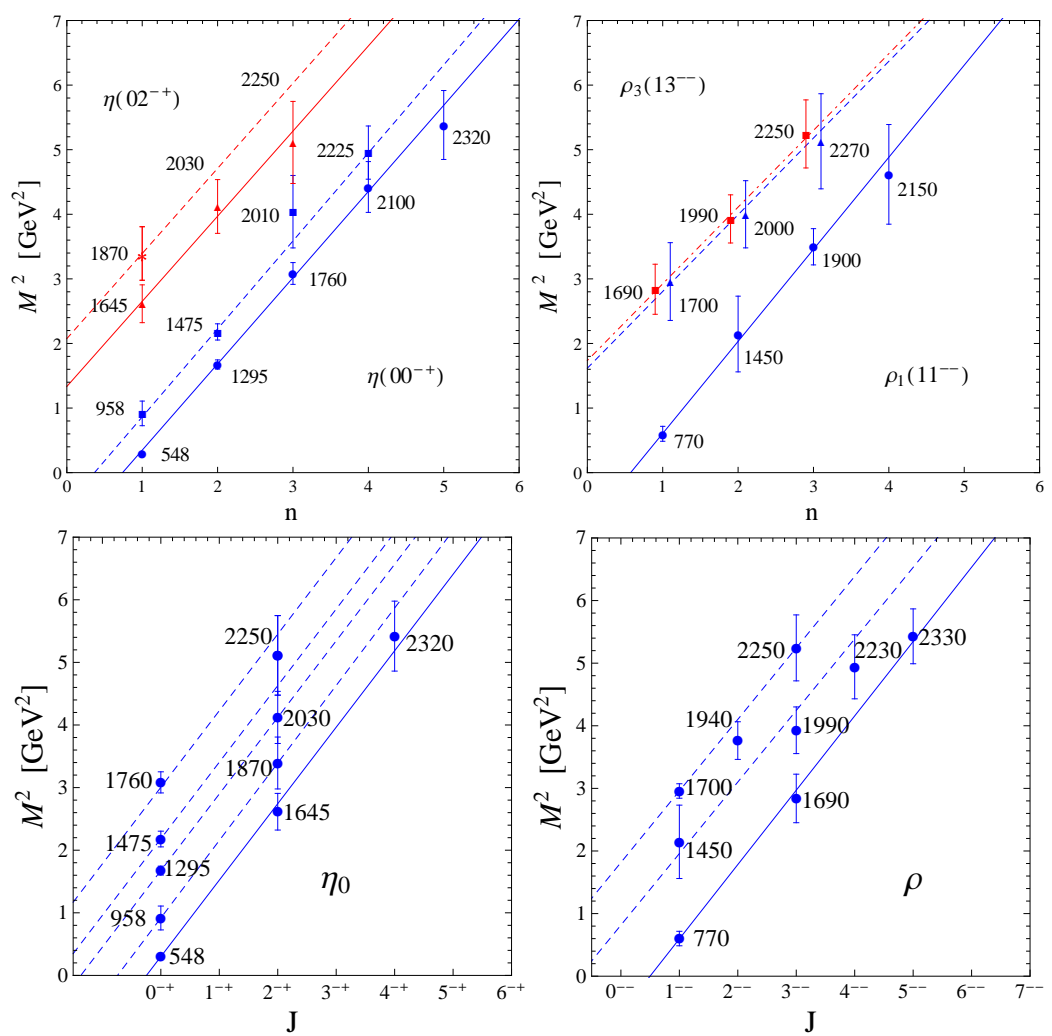

Fig. 1. The $\left(n, M^{2}\right)$ and $\left(J, M^{2}\right)$ plots for the $\eta$ and $\rho$ meson families.

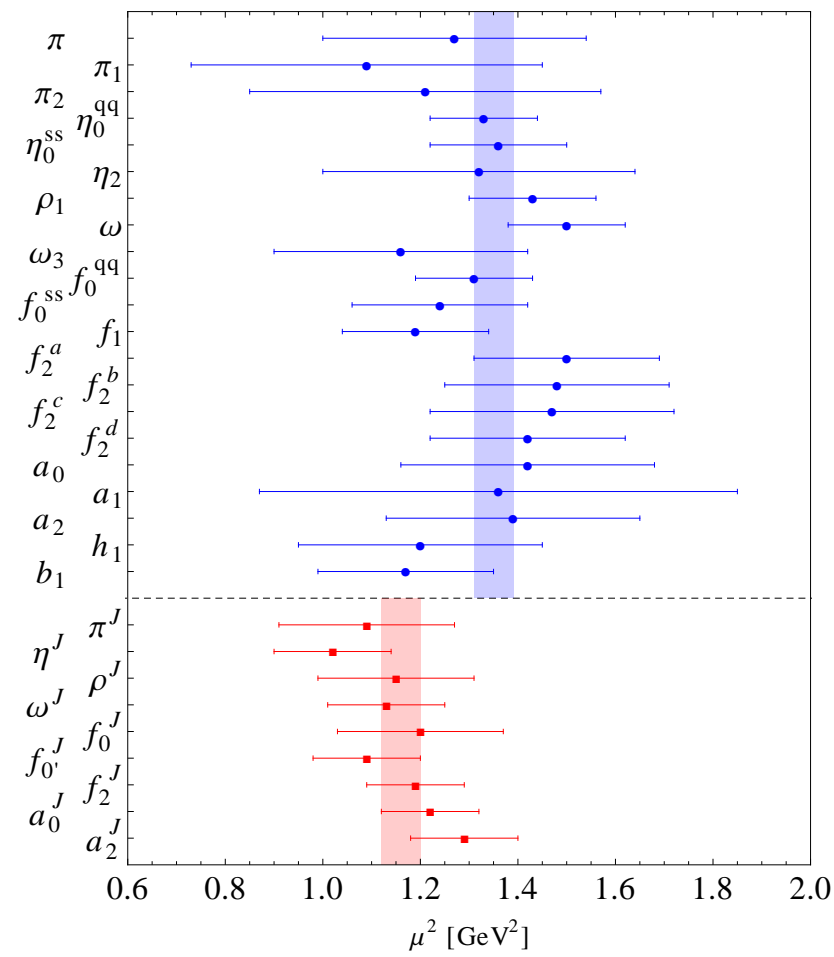

Fig. 2. $\left(n, M^{2}\right)$ and $\left(J, M^{2}\right)$ slopes for the meson families considered in Ref. [1]. 

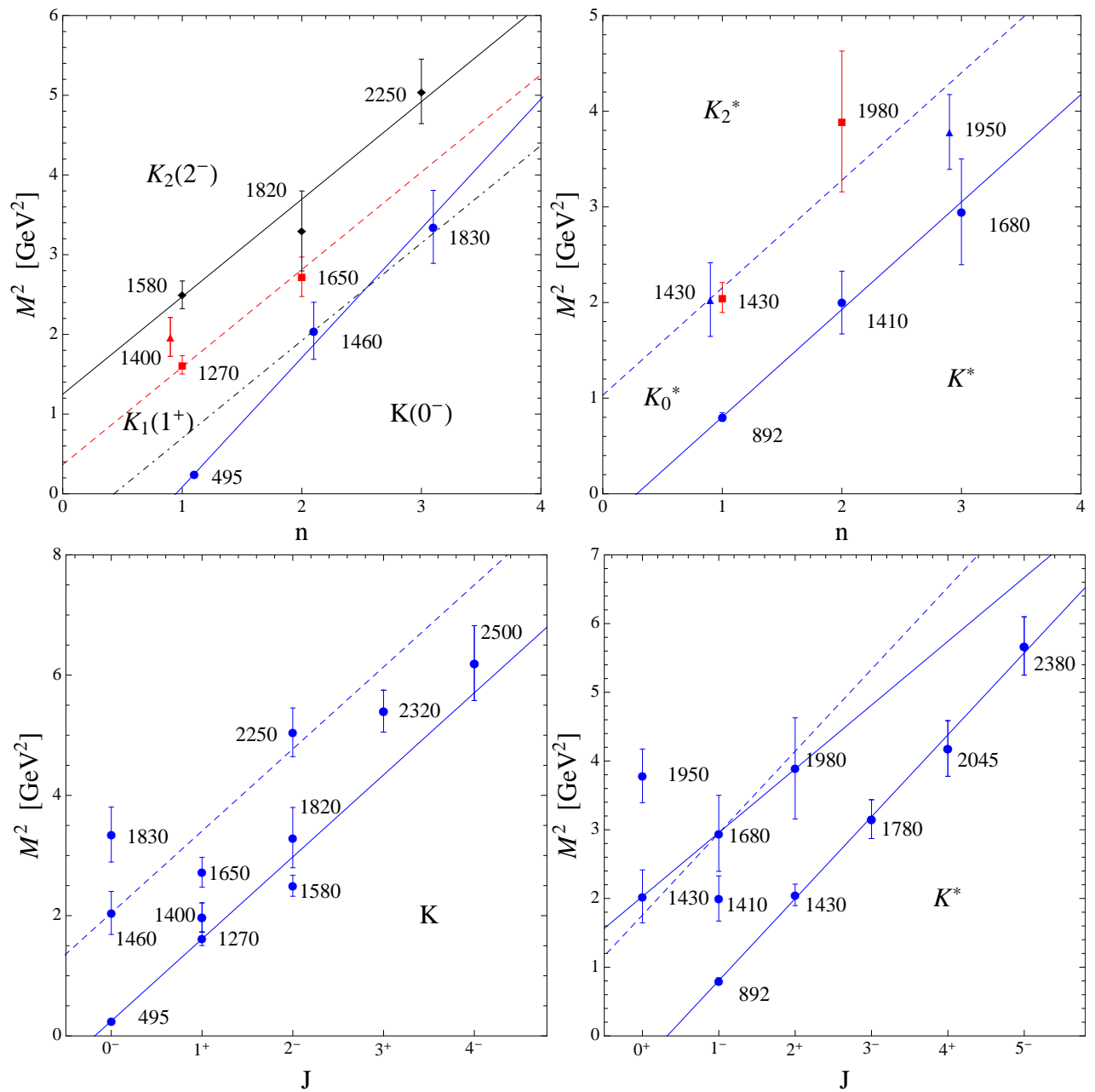

Fig. 3. $\left(n, M^{2}\right)$ and $\left(J, M^{2}\right)$ slopes for the kaon sector. The error bars follow from the half-width rule.

\section{References}

1. P. Masjuan, E. R. Arriola and W. Broniowski, Phys. Rev. D 85 (2012) 094006.

2. A. V. Anisovich, V. V. Anisovich and A. V. Sarantsev, Phys. Rev. D 62 (2000) 051502.

3. S. S. Afonin, Phys. Lett. B 639 (2006) 258.

4. K. Nakamura et al. [Particle Data Group Collaboration], J. Phys. G G 37 (2010) 075021.

5. E. Ruiz Arriola and W. Broniowski, Phys. Rev. D 81 (2010) 054009; E. Ruiz Arriola and W. Broniowski, (Bled Workshops in Physics. Vol. 12 No. 1), arXiv:1110.2863; P. Masjuan, S. Peris and J. J. Sanz-Cillero, Phys. Rev. D 78 (2008) 074028; P. Masjuan, arXiv:1206.2549. 